\title{
Second-refinement of Gauss-Seidel iterative method for solving linear system of equations
}

Tesfaye Kebede Enyew* , Gurju Awgichew, Eshetu Haile, Gashaye Dessalew Abie

Department of Mathematics, Bahir Dar University, Bahir Dar, Ethiopia

\begin{abstract}
Although large and sparse linear systems can be solved using iterative methods, its number of iterations is relatively large. In this case, we need to modify the existing methods in order to get approximate solutions in a small number of iterations. In this paper, the modified method called second-refinement of Gauss-Seidel method for solving linear system of equations is proposed. The main aim of this study was to minimize the number of iterations, spectral radius and to increase rate of convergence. The method can also be used to solve differential equations where the problem is transformed to system of linear equations with coefficient matrices that are strictly diagonally dominant matrices, symmetric positive definite matrices or M-matrices by using finite difference method. As we have seen in theorem land we assured that, if $\mathrm{A}$ is strictly diagonally dominant matrix, then the modified method converges to the exact solution. Similarly, in theorem 2 and 3 we proved that, if the coefficient matrices are symmetric positive definite or M-matrices, then the modified method converges. And moreover in theorem 4 we observed that, the convergence of second-refinement of Gauss-Seidel method is faster than Gauss-Seidel and refinement of Gauss-Seidel methods. As indicated in the examples, we demonstrated the efficiency of secondrefinement of Gauss-Seidel method better than Gauss-Seidel and refinement of GaussSeidel methods.
\end{abstract}

Keywords: Gauss-Seidel (GS) method, Refinement of Gauss-Seidel (RGS) method, Second- refinement of Gauss-Seidel (SRGS) method.

DOI: https://dx.doi.org/10.4314/ejst.v13i1.1

\section{INTRODUCTION}

Systems of linear equations arise in large number of areas both directly in modeling physical situations and indirectly in numerical solutions of other mathematical models. These applications occur in all areas of the physical, biological, social science, engineering, etc. (Laskar and Behera, 2014b). The

\footnotetext{
*Corresponding author: tk_ke@yahoo.com

(C) This is an Open Access article distributed under the terms of the Creative Commons Attribution License (http://creativecommons.org/licenses/CC BY4.0)
} 
solution of a system of equations can be obtained either by using direct method or iterative method. One of the earliest iterative methods is Gauss-Seidel iterative method named after the German mathematicians Carl Friedrich Gauss (1777 - 1855) and Philipp Ludwig von Seidel (1821 - 1896). The GaussSeidel method is also known as iteration by successive displacements or single steps by Geiringer and the Liebman method by Frankel. The convergence of the method is guaranteed for some special matrices such as strictly diagonally dominant (SDD), symmetric positive definite (SPD) or Mmatrices. In recent years, research results show that relaxation, generalized and refinement are used for modifying Gauss-Seidel method. The generalized Gauss-Seidel (GGS) method was developed by Salkuyeh (2007) and the researcher proved the convergence of the method by considering strictly diagonally dominant (SDD) matrices and M-matrices. The refinement of Gauss-Seidel (RGS) method was developed by Vatti and Tesfaye Kebede (2011) and who proved the convergence of the method by considering SDD matrices. The GGS method was also modified as refinement of GGS (RGGS) method (Laskar and Behera, 2014a) and considered SDD matrices to show the convergence of the method. All the above researchers tried to minimize the number of iterations and improved rate of convergence. But still different researchers have been conducting research in the area of iterative methods.

\section{METHODOLOGY}

Consider large and sparse linear systems of the form

$$
A x=b
$$

where $A=\left(a_{i j}\right)$ is nonsingular real matrix of order $n, b$ is a given $n$ dimensional real vector and $x$ is an $n$ dimensional vector to be determined. This equation can be computed by iterative methods based on splitting $A$ as:

$$
A=D-L-U
$$

where $D$ is a diagonal matrix with $a_{i i} \neq 0,-L$ and $-U$ are strictly lower and upper triangular parts of $A$, respectively (Varga, 1962; Saad, 2003). The coefficients matrices which we used in the examples were SDD, SPD, Mmatrices or a combination of these types. The convergence of the modified method was checked by using the number of iterations, spectral radius and rate of convergence.

The Gauss-Seidel iteration (GS) for first degree is

$$
x^{(n+1)}=(D-L)^{-1} U x^{(n)}+(D-L)^{-1} b
$$

If the matrix A is SDD, SPD and M-matrices, then equation (3) is convergent. 


\section{Refinement of Gauss-Seidel (RGS) Method}

Equation (1) could be solved by different methods. One of such methods is refinement of Gauss-Seidel (RGS) method. This method was introduced by Vatti and Tesfaye Kebede (2011). Now, use $A=D-L-U$ in equation (1), simplify and get the equation:

$$
\begin{aligned}
& x^{(n+1)}=\bar{x}^{(n+1)}+(D-L)^{-1}\left(b-A \bar{x}^{(n+1)}\right) . \text { Let us } \\
& \text { substitute } \bar{x}^{(n+1)}=(D-L)^{-1} U x^{(n)}+(D-L)^{-1} b \text {. Then we get } \\
& \begin{aligned}
x^{(n+1)}= & (D-L)^{-1} U x^{(n)}+(D-L)^{-1} b+(D-L)^{-1}\left[b-A\left((D-L)^{-1} U x^{(n)}+\right.\right. \\
& \left.\left.(D-L)^{-1} b\right)\right] \\
\Rightarrow x^{(n+1)}= & (D-L)^{-1} U x^{(n)}+(D-L)^{-1} b+(D-L)^{-1}[b-(D-L-U)( \\
& \left.\left.(D-L)^{-1} U x^{(n)}+(D-L)^{-1} b\right)\right] .
\end{aligned}
\end{aligned}
$$

After simplification, we obtain:

$$
x^{(n+1)}=\left[(D-L)^{-1} U\right]^{2} x^{(n)}+\left[I+(D-L)^{-1} U\right](D-L)^{-1} b .
$$

Equation (4) is called refinement of Gauss-Seidel (RGS) method.

If A is SDD, SPD and M-matrices, then the refinement of Gauss-Seidel method converges to exact solution.

\section{Second-Refinement of Gauss-Seidel (SRGS) Method}

Equation (1) can be rewritten as:

$$
\begin{aligned}
& (D-L-U) x=b \quad \Rightarrow(D-L) x=U x+b \\
& \Rightarrow(D-L) x=(D-L-A) x+b, \text { where } U=D-L-A \\
& \Rightarrow(D-L) x=(D-L) x+b-A x \quad \Rightarrow x=x+(D-L)^{-1}(b-A x) \\
& \Rightarrow x^{(n+1)}=\bar{x}^{(n+1)}+(D-L)^{-1}\left(b-A \bar{x}^{(n+1)}\right) .
\end{aligned}
$$

By taking $x^{(n+1)}=\bar{x}^{(n+1)}+(D-L)^{-1}\left(b-A \bar{x}^{(n+1)}\right)$ substitute equation (4) on $\bar{x}^{(n+1)}$ in the right side of this equation, we get:

$$
\begin{aligned}
\Rightarrow x^{(n+1)}= & {\left[(D-L)^{-1} U\right]^{2} x^{(n)}+\left[I+(D-L)^{-1} U\right](D-L)^{-1} b+(D-L)^{-1} } \\
& \left(b-A\left\{\left[(D-L)^{-1} U\right]^{2} x^{(n)}+\left[I+(D-L)^{-1} U\right](D-L)^{-1} b\right\}\right) .
\end{aligned}
$$


After simplifying the above equation, we get:

$$
x^{(n+1)}=\left[(D-L)^{-1} U\right]^{3} x^{(n)}+\left\lfloor I+(D-L)^{-1} U+\left((D-L)^{-1} U\right)^{2}\right\rfloor(D-L)^{-1} b
$$

Equation (5) is called second-refinement of Gauss-Seidel (SRGS) method.

\section{Convergence of Second-Refinement of Gauss-Seidel (SRGS) Method}

Theorem 1: If $\mathrm{A}$ is strictly diagonally dominant (SDD) matrix, then the second-refinement of Gauss-Seidel (SRGS) method is convergent for any initial guess $x^{(0)}$.

\section{Proof:}

A similar proof as this theorem can be obtained in (Dafchahi, 2008; Vatti and Genanew Gofe, 2011; Vatti and Tesfaye Kebede, 2011; Laskar and Behera, 2014a).

Let $X$ be the real exact solution of (1). Given that A is SDD matrix. Then GS and RGS methods are convergent and so let $\bar{x}^{(n+1)}$ converges to $X$ when

$$
\begin{aligned}
& \bar{x}^{(n+1)}=\left[(D-L)^{-1} U\right]^{2} x^{(n)}+\left[I+(D-L)^{-1} U\right](D-L)^{-1} b . \text { Then, } \\
& x^{(n+1)}=\bar{x}^{(n+1)}+(D-L)^{-1}\left(b-A \bar{x}^{(n+1)}\right) \text { or } \\
& x^{(n+1)}-X=\bar{x}^{(n+1)}-X+(D-L)^{-1}\left(b-A \bar{x}^{(n+1)}\right) .
\end{aligned}
$$

Hence, by taking norm to both sides

$$
\begin{gathered}
\left\|x^{(n+1)}-X\right\|=\| \begin{array}{l}
\bar{x}^{(n+1)}-X+(D-L)^{-1}\left(b-A \bar{x}^{(n+1)}\right)\|\leq\| \bar{x}^{(n+1)}-X \|+ \\
\left\|(D-L)^{-1}\left(b-A \bar{x}^{(n+1)}\right)\right\|
\end{array} \\
\Rightarrow\left\|x^{(n+1)}-X\right\| \leq\left\|\bar{x}^{(n+1)}-X\right\|+\left\|(D-L)^{-1}\right\|\left\|\left(b-A \bar{x}^{(n+1)}\right)\right\| \rightarrow\|X-X\|+ \\
\left\|(D-L)^{-1}\right\|\|b-A X\| \\
=0+\left\|(D-L)^{-1}\right\| b-b \|=0+0=0 .
\end{gathered}
$$

Hence, $x^{(n+1)}$ converges to $X$.

$$
\Rightarrow \rho\left(\left((D-L)^{-1} U\right)^{3}\right)=\rho\left((D-L)^{-1} U\right)^{3}<1 \text {. }
$$

Therefore, the SRGS method is convergent. 
Theorem 2: If A is symmetric positive definite matrix (SPD), then the secondrefinement of Gauss-Seidel (SRGS) method converges for any arbitrary choice of the initial approximation $x^{(0)}$.

\section{Proof:}

According to Quarteroni et al. (2000), we can prove this theorem by using consistency and spectral radius of the iterative matrix or definition of convergence of the method. Given equation (3), then we have $\rho\left((D-L)^{-1} U\right)<1$ since A is SPD matrix. Let $X$ be the exact solution of (1). Then the Gauss-Seidel iterative method can be rewritten as: $X=\left[I-(D-L)^{-1} U\right]^{-1}(D-L)^{-1} b$. Using equation (5) we have: $x^{(n+1)}=\left[(D-L)^{-1} U\right]^{3} x^{(n)}+\left\lfloor I+(D-L)^{-1} U+\left((D-L)^{-1} U\right)^{2}\right\rfloor(D-L)^{-1} b$

First, we have to check consistency of SRGS method with GS method.

From GS method, we have $\left\|(D-L)^{-1} U\right\|<1 \Rightarrow \rho\left((D-L)^{-1} U\right)<1$.

After substituting $X$ in equation (5), one can get,

$$
\begin{aligned}
X= & \left((D-L)^{-1} U\right)^{3} X+\left[I+(D-L)^{-1} U+\left((D-L)^{-1} U\right)^{2}\right](D-L)^{-1} b \\
\Rightarrow & X=\left(I-\left((D-L)^{-1} U\right)^{3}\right)^{-1}\left[I+(D-L)^{-1} U+\left((D-L)^{-1} U\right)^{2}\right](D-L)^{-1} b \\
= & {\left[I+\left((D-L)^{-1} U\right)^{3}+\left((D-L)^{-1}\right)^{6}+\ldots . .\right]\left[I+(D-L)^{-1} U+\right.} \\
& \left.\left((D-L)^{-1} U\right)^{2}\right](D-L)^{-1} b
\end{aligned}
$$

Since $(\mathrm{I}-\mathrm{M})^{-1}=\mathrm{I}+\mathrm{M}+\mathrm{M}^{2}+\ldots \quad$ if $\rho(\mathrm{M})<1$ and $\mathrm{I}-\mathrm{M}$ is nonsingular.

$$
\begin{aligned}
& \quad \begin{array}{l}
\quad \\
\quad \\
\quad+\ldots](D-L)^{-1} b
\end{array} \\
& =\left[I-(D-L)^{-1} U\right]^{-1}(D-L)^{-1} b . \text { Since }(\mathrm{I}-\mathrm{M})^{-1}=\mathrm{I}+\mathrm{M}+\mathrm{M}^{2}+\ldots \\
& \therefore X=\left[I-(D-L)^{-1} U\right]^{-1}(D-L)^{-1} b \text { is consistent to Gauss-Seidel } \\
& \text { method. }
\end{aligned}
$$

Next, let us check convergence of the modified method or SRGS method when $A$ is SPD matrix. 


$$
\begin{aligned}
\hline x^{(n+1)}= & {\left[(D-L)^{-1} U\right]^{3} x^{(n)}+\left[I+(D-L)^{-1} U+\left((D-L)^{-1} U\right)^{2}\right](D-L)^{-1} b } \\
= & {\left[(D-L)^{-1} U\right]^{6} x^{(n-1)}+\left[I+(D-L)^{-1} U+\left((D-L)^{-1} U\right)^{2}+\left((D-L)^{-1} U\right)^{3}+\right.} \\
& \left.\left((D-L)^{-1} U\right)^{4}+\left((D-L)^{-1} U\right)^{5}\right](D-L)^{-1} b \\
= & {\left[(D-L)^{-1} U\right]^{9} x^{(n-2)}+\left[I+(D-L)^{-1} U+\left((D-L)^{-1} U\right)^{2}+\ldots+\right.} \\
& \left.\left((D-L)^{-1} U\right)^{8}\right](D-L)^{-1} b
\end{aligned}
$$

and so on. For $\mathrm{n}=0,1,2, \ldots$

$$
\begin{aligned}
= & {\left[(D-L)^{-1} U\right]^{3 n+3} x^{(0)}+\left[I+(D-L)^{-1} U+\left((D-L)^{-1} U\right)^{2}+\right.} \\
& \left.\left((D-L)^{-1} U\right)^{3}+\ldots+\left((D-L)^{-1} U\right)^{3 n+2}\right](D-L)^{-1} b
\end{aligned}
$$

Hence, $\rho\left((D-L)^{-1} U\right)<1$ since A is SPD.

$$
\begin{aligned}
& \Rightarrow \lim _{n \rightarrow \infty}\left[(D-L)^{-1} U\right]^{3 n+3} x^{(0)}=0 \\
& \Rightarrow \lim _{n \rightarrow \infty} x^{(n+1)}=\lim _{n \rightarrow \infty}\left[(D-L)^{-1} U\right]^{3 n+3}+\lim _{n \rightarrow \infty} \sum_{k=0}^{3 n+2}\left((D-L)^{-1} U\right)^{k}(D-L)^{-1} b \\
& =0+\left(I-(D-L)^{-1} U\right)^{-1}(D-L)^{-1} b \\
& =\left(I-(D-L)^{-1} U\right)^{-1}(D-L)^{-1} b=X . \Rightarrow x^{(n+1)} \rightarrow X . \\
& \Rightarrow \rho\left\lfloor\left((D-L)^{-1} U\right)^{3}\right]=\left[\rho\left((D-L)^{-1} U\right)\right]^{3}<1 \text {. }
\end{aligned}
$$

Or we can show by using spectral radius of the iteration matrix since A is SPD matrix. Therefore, the second-refinement of Gauss-Seidel method is convergent.

Theorem 3: If $A$ is an M-matrix, then the second-refinement of Gauss-Seidel (SRGS) iterative method converges for any initial guess $x^{(0)}$.

\section{Proof:}

From theorem 2, we proved that our modified method (SRGS method) is consistent with GS method. So, we can show the convergence of the method by using spectral radius of iterative matrix.If the matrix $\mathrm{A}$ is an M-matrix, then the spectral radius of GS is less than 1. Thus, $\rho\left(\left((D-L)^{-1} U\right)\right)<1$.

$\Rightarrow \rho\left(\left((D-L)^{-1} U\right)^{3}\right)=\left[\rho\left((D-L)^{-1} U\right)\right]^{3}<1$. The spectral radius of SRGS method is less than 1 .

$\therefore$ SRGS method is convergent. 
Theorem 4: The second-refinement of Gauss-Seidel method converges faster than the Gauss-Seidel method and refinement of Gauss-Seidel method when Gauss-Seidel method is convergent.

\section{Proof:}

We can rewrite equation (3), (4) and (5), respectively, by $x^{(n+1)}=G x^{(n)}+C$, $x^{(n+1)}=G^{2} x^{(n)}+B$ and $x^{(n+1)}=G^{3} x^{(n)}+K$ where $G=(D-L)^{-1} U$, $C=(D-L)^{-1} b, B=\left[I+(D-L)^{-1} U\right](D-L)^{-1} b$ and $K=\left\lfloor I+(D-L)^{-1} U+\left((D-L)^{-1} U\right)^{2}\right\rfloor(D-L)^{-1} b$. Given that $\|G\|<1$.

Let $X$ be the exact solution of (1).

$\Rightarrow X=G X+C, X=G^{2} X+B$ and $X=G^{3} X+K$. And let $n=0,1,2, \ldots$ be nonnegative integer.

If we consider Gauss-Seidel method:

$$
\begin{aligned}
& x^{(n+1)}=G x^{(n)}+C \Rightarrow x^{(n+1)}-X=G x^{(n)}-X+C \\
& \begin{aligned}
& \Rightarrow x^{(n+1)}-X=G\left(x^{(n)}-X\right)+G X+C-X \\
& \Rightarrow x^{(n+1)}-X=G\left(x^{(n)}-X\right) \\
& \Rightarrow\left\|x^{(n+1)}-X\right\|\left\|G\left(x^{(n)}-X\right)\right\| \leq\|G\|\left\|x^{(n)}-X\right\| \leq\left\|G^{2}\right\|\left\|x^{(n-1)}-X\right\| \leq \ldots \\
& \leq\left\|G^{n}\right\|\left\|x^{(1)}-X\right\| \\
& \Rightarrow\left\|x^{(n+1)}-X\right\| \leq\left\|G^{n}\right\| x^{(1)}-X\|\leq\| G\left\|^{n}\right\| x^{(1)}-X \| .
\end{aligned}
\end{aligned}
$$

Now, let us consider refinement of Gauss-Seidel method.

$$
\begin{aligned}
& x^{(n+1)}=G^{2} x^{(n)}+B \Rightarrow x^{(n+1)}-X=G^{2} x^{(n)}-X+B \\
& \begin{array}{c}
\Rightarrow x^{(n+1)}-X=G^{2}\left(x^{(n)}-X\right)+G^{2} X+B-X \\
\Rightarrow\left\|x^{(n+1)}-X\right\|=\left\|G^{2}\left(x^{(n)}-X\right)\right\| \leq\left\|G^{2}\right\|\left\|x^{(n)}-X\right\| \leq\left\|G^{4}\right\|\left\|x^{(n-1)}-X\right\| \leq \ldots \\
\quad \leq\left\|G^{2 n}\right\|\left\|x^{(1)}-X\right\|
\end{array} \\
& \Rightarrow\left\|x^{(n+1)}-X\right\| \leq\left\|G^{2 n}\right\|\left\|x^{(1)}-X\right\| \leq\|G\|^{2 n}\left\|x^{(1)}-X\right\| .
\end{aligned}
$$


Again let us consider second-refinement of Gauss-Seidel method.

$$
\begin{aligned}
& x^{(n+1)}=G^{3} x^{(n)}+K \Rightarrow x^{(n+1)}-X=G^{3} x^{(n)}-X+K \\
& \begin{aligned}
& \Rightarrow x^{(n+1)}-X=G^{3}\left(x^{(n)}-X\right)+G^{3} X+K-X \\
& \Rightarrow\left\|x^{(n+1)}-X\right\|=\left\|G^{3}\left(x^{(n)}-X\right)\right\| \leq\left\|G^{3}\right\|\left\|x^{(n)}-X\right\| \leq\left\|G^{6}\right\|\left\|x^{(n-1)}-X\right\| \leq \ldots \\
& \quad \quad\left\|G^{3 n}\right\| x^{(1)}-X \|
\end{aligned} \\
& \Rightarrow\left\|x^{(n+1)}-X\right\| \leq\left\|G^{3 n}\right\|\left\|x^{(1)}-X\right\| \leq\|G\|^{3 n}\left\|x^{(1)}-X\right\| .
\end{aligned}
$$

According to the coefficients of above inequalities, we have

$$
\|G\|^{3 n}<\|G\|^{2 n}<\|G\|^{n} \text { since }\|G\|<1 \text {. }
$$

Therefore, the second-refinement of Gauss-Seidel method converges faster than the Gauss-Seidel method and refinement of Gauss-Seidel method.

\section{$M^{\text {th }}$-Refinement of Gauss-Seidel Method}

We can generalize the refinement of Gauss-Seidel method by $\mathrm{m}^{\text {th }}$-refinement of Gauss-Seidel iterative method. This general form of RGS method can be derived by considering the following patterns.

$>$ Refinement of Gauss-Seidel method is

$$
x^{(n+1)}=\left[(D-L)^{-1} U\right]^{2} x^{(n)}+\left[I+(D-L)^{-1} U\right](D-L)^{-1} b,
$$

$>$ Second-refinement of Gauss-Seidel method is

$$
x^{(n+1)}=\left[(D-L)^{-1} U\right]^{\beta} x^{(n)}+\left\lfloor I+(D-L)^{-1} U+\left((D-L)^{-1} U\right)^{2}\right\rfloor(D-L)^{-1} b
$$

$>$ Third-refinement of Gauss-Seidel method is

$$
\begin{aligned}
x^{(n+1)}= & {\left[(D-L)^{-1} U\right]^{4} x^{(n)}+\left[I+(D-L)^{-1} U+\left((D-L)^{-1} U\right)^{2}+\right.} \\
& \left.\left((D-L)^{-1} U\right)^{3}\right](D-L)^{-1} b
\end{aligned}
$$

$>$ Fourth-refinement of Gauss-Seidel method is

$$
\begin{aligned}
x^{(n+1)}= & {\left[(D-L)^{-1} U\right]^{5} x^{(n)}+\left[I+(D-L)^{-1} U+\left((D-L)^{-1} U\right)^{2}+\right.} \\
& \left.\left((D-L)^{-1} U\right)^{3}+\left((D-L)^{-1} U\right)^{4}\right](D-L)^{-1} b
\end{aligned}
$$

and so on

The $\mathrm{m}^{\text {th }}$-refinement of Gauss-Seidel method is

$$
\begin{aligned}
x^{(n+1)}= & {\left[(D-L)^{-1} U\right]^{n+1} x^{(n)}+\left[I+(D-L)^{-1} U+\left((D-L)^{-1} U\right)^{2}+\ldots+\right.} \\
& \left.\left((D-L)^{-1} U\right)^{m}\right](D-L)^{-1} b
\end{aligned}
$$

where $\mathrm{m}=0,1,2,3, \ldots$ 


$$
\begin{aligned}
& \Rightarrow x^{(n+1)}=\left[(D-L)^{-1} U\right]^{m+1} x^{(n)}+\left[\sum_{k=0}^{m}\left((D-L)^{-1} U\right)^{k}\right](D-L)^{-1} b \\
& \Rightarrow x^{(n+1)}=\left[(D-L)^{-1} U\right]^{m+1} x^{(n)}+\left(I-(D-L)^{-1} U\right)^{-1}(D-L)^{-1} b \text {. Since } \\
& \rho\left((D-L)^{-1} U\right)<1 \\
& \Rightarrow x^{(n+1)}=\left[(D-L)^{-1} U\right]^{m+1} x^{(n)}+\left(I-(D-L)^{-1} U\right)^{-1}(D-L)^{-1} b
\end{aligned}
$$

where $\rho\left((D-L)^{-1} U\right)<1$ and $m \rightarrow \infty$

$$
\therefore x^{(n+1)}=\left[(D-L)^{-1} U\right]^{n+1} x^{(n)}+\left[\sum_{k=0}^{m}\left((D-L)^{-1} U\right)^{k}\right](D-L)^{-1} b \text {. }
$$

Equation (6) is called $\mathrm{m}^{\text {th }}$-refinement of Gauss-Seidel method.

If $m=0$ the scheme is equivalent to Gauss-Seidel method.

If $m=1$ the method is equivalent to refinement of Gauss-Seidel method.

If $\mathrm{m}=2$ the scheme is equivalent to second-refinement of Gauss-Seidel method, and so on.

\section{Numerical Examples}

Example 1: Consider the following system of linear equations whose coefficient matrix is both SDD and SPD with tolerance 0.0001.

$$
\left\{\begin{array}{c}
6 x_{1}+2 x_{2}+2 x_{3}=5 \\
2 x_{1}+8 x_{2}+2 x_{3}=6 \\
2 x_{1}+2 x_{2}+10 x_{3}=7
\end{array}\right.
$$

Solution: From table 1, one can see that the spectral radius of SRGS method is 0.0021 which is smaller than spectral radius of GS and RGS methods 0.1291 and 0.0167 respectively. As presented in Table 2, the exact solution for the given linear system of equations is $(0.5,0.5,0.5)$. It is observed from the table that the same solution is obtained by using Gauss-Seidel method (GS) at the $6^{\text {th }}$ iteration, refinement of Gauss-Seidel method (RGS) at the $3^{\text {rd }}$ iteration and second refinement of Gauss-Seidel (SRGS) method at the $2^{\text {nd }}$ iteration. Rate of convergence for GS, RGS and SRGS are 2.0472, 4.0923 and 6.1658, respectively.

Example 2: Consider the following system of linear equations whose coefficient matrix is SDD but not SPD with tolerance 0.0001 . 


$$
\left\{\begin{array}{c}
6 x_{1}+4 x_{2}-x_{3}=9 \\
3 x_{1}+7 x_{2}+2 x_{3}=12 \\
-4 x_{1}+3 x_{2}+8 x_{3}=7
\end{array}\right.
$$

Table 1. Comparsion of the spectral radius, CPU time and iteration number.

\begin{tabular}{lccc}
\hline Method & GS & RGS & SRGS \\
\hline Spectral radius & 0.1291 & 0.0167 & 0.0021 \\
CPU time (second) & 36.126 & 33.107 & 32.931 \\
Iteration number & 6 & 3 & 2 \\
\hline
\end{tabular}

Table 2. Numerical results of example 1 and comparison between GS, RGS and SRGS.

\begin{tabular}{rrrrrrrrrr}
\hline & \multicolumn{4}{c}{$\mathbf{G S}$} & \multicolumn{4}{c}{$\mathbf{R G S}$} & \multicolumn{3}{c}{ SRGS } \\
\hline $\mathbf{n}$ & $\mathbf{x}_{\mathbf{1}}{ }^{(\mathbf{n})}$ & $\mathbf{x}_{\mathbf{2}}{ }^{(\mathbf{n})}$ & $\mathbf{x}_{\mathbf{3}}{ }^{(\mathbf{n})}$ & $\mathbf{x}_{\mathbf{1}}{ }^{(\mathbf{n})}$ & $\mathbf{x}_{\mathbf{2}}{ }^{(\mathbf{n}}$ & \multicolumn{1}{c}{$\mathbf{x}_{\mathbf{3}}{ }^{(\mathbf{n})}$} & $\mathbf{x}_{\mathbf{1}}{ }^{(\mathbf{n})}$ & $\mathbf{x}_{\mathbf{2}}{ }^{(\mathbf{n})}$ & $\mathbf{x}_{\mathbf{3}}{ }^{(\mathbf{n})}$ \\
\hline 0 & 0.0000 & 0.0000 & 0.0000 & 0.0000 & 0.0000 & 0.0000 & 0.0000 & 0.0000 & 0.0000 \\
1 & 0.8333 & 0.5417 & 0.4250 & 0.5111 & 0.5160 & 0.4946 & 0.4965 & 0.5022 & 0.5003 \\
2 & 0.5111 & 0.5160 & 0.4946 & 0.4992 & 0.5001 & 0.5001 & 0.5000 & 0.5000 & 0.5000 \\
3 & 0.4965 & 0.5022 & 0.5003 & 0.5000 & 0.5000 & 0.5000 & & & \\
4 & 0.4992 & 0.5001 & 0.5001 & & & & & & \\
5 & 0.4999 & 0.5000 & 0.5000 & & & & & & \\
6 & 0.5000 & 0.5000 & 0.5000 & & & & &
\end{tabular}

Solution:Table 3 shows that the spectral radius of SRGS method is 0.2741 which is less than the other two methods GS and RGS whose spectral radius are 0.6496 and 0.4220 respetively. Which means that the modified method SRGS is faster than GS and RGS method. The exact solution for the above equation is $(1,1,1)$. Table 4 shows that this solution is obtained after $16^{\text {th }}$ iteration by using GS method, $9^{\text {th }}$ iteration by using RGS method and $7^{\text {th }}$ iterations by using SRGS method.Rate of convergence for GS, RGS and SRGS are 0.4314, 0.8627 and 1.2943 , respectively.

Table 3. Comparison of the spectral radius, CPU time and iteration number.

\begin{tabular}{llcc}
\hline Method & GS & RGS & SRGS \\
\hline Spectral radius & 0.6496 & 0.4220 & 0.2741 \\
CPU time (second) & 46.433 & 39.921 & 38.463 \\
Iteration number & 16 & 9 & 7 \\
\hline
\end{tabular}

Example 3: Consider the following system of linear equations whose coefficient matrix is SPD but not SDD with tolerance 0.0001 .

$$
\left\{\begin{array}{l}
6 x_{1}+4 x_{2}+3 x_{3}=13 \\
4 x_{1}+5 x_{2}+2 x_{3}=11 \\
3 x_{1}+2 x_{2}+2 x_{3}=7
\end{array}\right.
$$


Solution:Table 5 shows that the spectral radius of SRGS method is 0.4219 which is less than the other two methods GS and RGS whose spectral radius are 0.7500 and 0.5625 , respectively. Which means that the modified method SRGS is faster than GS and RGS method. The exact solution for the above equation is $(1,1,1)$. Table 6 shows that this solution is obtained at $38^{\text {th }}$ iteration by using GS method, $20^{\text {th }}$ iteration by using RGS method and $17^{\text {th }}$ iterations by using SRGS method.Rate of convergence for GS, RGS and SRGS are 0.2877, 0.5754 and 0.8630 , respectively.

Table 4. Numerical results of example 2 and comparison between GS, RGS and SRGS.

\begin{tabular}{|c|c|c|c|c|c|c|c|c|c|c|}
\hline & \multicolumn{3}{|c|}{ GS } & \multicolumn{3}{|c|}{ RGS } & \multicolumn{4}{|c|}{ SRGS } \\
\hline $\mathbf{n}$ & $\mathbf{x}_{1}{ }^{(n)}$ & $\mathbf{x}_{2}{ }^{(\mathrm{n})}$ & $\mathbf{x}_{3}{ }^{(\mathbf{n})}$ & $\mathbf{x}_{1}^{(\mathrm{n})}$ & $\mathbf{x}_{2}^{\left({ }^{(n)}\right.}$ & $\overline{\mathbf{x}_{3}{ }^{(\mathbf{n})}}$ & $\overline{\mathbf{x}_{1}{ }^{(\mathbf{n})}}$ & $\mathbf{x}_{2}$ & (n) & $\mathbf{X}_{3}{ }^{(\mathbf{n})}$ \\
\hline 0 & 0.0000 & 0.0000 & 0.0000 & 0.0000 & 0.0000 & 0.0000 & 0.0000 & 0.0000 & 0.000 & \\
\hline 1 & 1.5000 & 1.0714 & 1.2232 & 0.9896 & 0.9407 & 1.0170 & 1.0424 & 0.9770 & 1.0298 & \\
\hline 2 & 0.9896 & 0.9407 & 1.0170 & 1.0203 & 0.9828 & 1.0166 & 1.0091 & 0.9929 & 1.0072 & \\
\hline 3 & 1.0424 & 0.9770 & 1.0298 & 1.0091 & 0.9929 & 1.0072 & 1.0025 & 0.9981 & 1.0020 & \\
\hline .. & $\ldots$ & $\ldots$ & $\ldots$ & & & & & $\ldots$ & & \\
\hline 7 & 1.0059 & 0.9954 & 1.0047 & 1.0003 & 0.9998 & 1.0002 & 1.0000 & 1.0000 & 1.0000 & \\
\hline 8 & 1.0039 & 0.9970 & 1.0030 & 1.0001 & 0.9999 & 1.0001 & & & & \\
\hline 9 & 1.0025 & 0.9981 & 1.0020 & 1.0001 & 1.0000 & 1.0000 & & & & \\
\hline 10 & 1.0016 & 0.9987 & 1.0013 & & & & & & & \\
\hline.. & $\ldots$ & $\ldots$ & $\ldots$ & & & & & & & \\
\hline 16 & 1.0001 & 0.9999 & 1.0001 & & & & & & & \\
\hline
\end{tabular}

Table 5. Comparison of the spectral radius, CPU time and iteration number.

\begin{tabular}{lccc}
\hline Method & GS & RGS & SRGS \\
\hline Spectral radius & 0.7500 & 0.5625 & 0.4219 \\
CPU time (second) & 49.281 & 40.819 & 35.553 \\
Iteration number & 38 & 20 & 13 \\
\hline
\end{tabular}

Example 4: Consider the following system of linear equations whose coefficient matrix is SDD but not PD and SPD with tolerance 0.0001 .

$$
\left\{\begin{array}{l}
5 x_{1}+3 x_{2}+x_{3}=9 \\
4 x_{1}-6 x_{2}+x_{3}=-1 \\
2 x_{1}+x_{2}+4 x_{3}=7
\end{array}\right.
$$

Solution:Table 7 shows that the spectral radius of SRGS method is 0.0772 which is less than the other two methods GS and RGS methods whose spectral radius are 0.4258 and 0.1813 , respectively. The exact solution of the system is $(1,1,1)$. From table 8 one can see that the solution is obtained after 12 iterations by using GS method, 6 iterations by using RGS method and 4 iterations by using SRGS method.Rate of convergence for GS, RGS and SRGS are $0.8538,1.7076$ and 2.5614 , respectively. 
Table 6. Numerical results of example 3 and comparison between GS, RGS and SRGS.

\begin{tabular}{|c|c|c|c|c|c|c|c|c|c|}
\hline \multicolumn{4}{|c|}{ GS } & \multicolumn{3}{|c|}{ RGS } & \multicolumn{3}{|c|}{ SRGS } \\
\hline $\mathbf{n}$ & $\mathbf{x}_{1}{ }^{(\mathbf{n})}$ & $\mathbf{x}_{2}{ }^{(\mathbf{n})}$ & $\mathbf{x}_{3}{ }^{(n)}$ & $\mathbf{x}_{1}{ }^{(n)}$ & $\mathbf{X}_{2}{ }^{(\mathbf{n})}$ & $\mathbf{X}_{3}{ }^{(\mathbf{n})}$ & $\mathbf{x}_{1}{ }^{(\mathbf{n})}$ & $\mathbf{X}_{2}{ }^{(\mathbf{n})}$ & $\mathbf{x}_{3}{ }^{(\mathbf{n})}$ \\
\hline 0 & 0.0000 & 0.0000 & 0.0000 & 0.0000 & 0.0000 & 0.0000 & 0.0000 & 0.0000 & 0.0000 \\
\hline 1 & 2.1667 & 0.4667 & -0.2167 & 1.9639 & 0.7156 & -0.1614 & 1.7703 & 0.8483 & -0.0038 \\
\hline 2 & 1.9639 & 0.7156 & -0.1614 & 1.6030 & 0.9191 & 0.1764 & 1.3565 & 0.9770 & 0.4883 \\
\hline 3 & 1.7703 & 0.8483 & -0.0038 & 1.3565 & 0.9770 & 0.4883 & 1.1552 & 0.9965 & 0.7707 \\
\hline .. & $\ldots$ & $\ldots$ & $\ldots$ & $\ldots$ & $\ldots$ & $\ldots$ & $\ldots$ & $\ldots$ & \\
\hline 13 & 1.0497 & 0.9997 & 0.9257 & 1.0012 & 1.0000 & 0.9982 & 1.0000 & 1.0000 & 1.0000 \\
\hline .. & $\ldots$ & $\ldots$ & $\ldots$ & $\ldots$ & $\ldots$ & ... & & & \\
\hline 20 & 1.0067 & 1.0000 & 0.9900 & 1.0000 & 1.0000 & 1.000 & & & \\
\hline$\ddot{3} 8$ & 1.0000 & 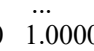 & $\begin{array}{ll}\cdots \\
0 & 1.0000\end{array}$ & & & & & & \\
\hline
\end{tabular}

Table 7. Comparison of the Spectral Radius, CPU time and iteration number.

\begin{tabular}{llll}
\hline Method & GS & RGS & SRGS \\
\hline Spectral radius & 0.4258 & 0.1813 & 0.0772 \\
CPU time (second) & 49.533 & 42.356 & 41.488 \\
Iteration number & 12 & 6 & 4 \\
\hline
\end{tabular}

Example 5: Consider the following system of linear equations whose coefficient matrix is an M-matrix $A$ (or 2-cyclic matrix $A$ ), which arises from the discretization of the Poisson's equation $\frac{\partial^{2} T}{\partial x^{2}}+\frac{\partial^{2} T}{\partial y^{2}}=f$ on the unit square as considered by (Dafchahi, 2008; Datta , 2010; Vatti and GenanewGofe, 2011), with tolerance 0.00001 .

$$
\left(\begin{array}{rrrrrr}
4 & -1 & 0 & -1 & 0 & 0 \\
-1 & 4 & -1 & 0 & -1 & 0 \\
0 & -1 & 4 & 0 & 0 & -1 \\
-1 & 0 & 0 & 4 & -1 & 0 \\
0 & -1 & 0 & -1 & 4 & -1 \\
0 & 0 & -1 & 0 & -1 & 4
\end{array}\right)\left(\begin{array}{l}
x_{1} \\
x_{2} \\
x_{3} \\
x_{4} \\
x_{5} \\
x_{6}
\end{array}\right)=\left(\begin{array}{l}
1 \\
0 \\
0 \\
0 \\
0 \\
0
\end{array}\right)
$$

Solution:Table 9 shows that the spectral radius of SRGS method is 0.0483 which is less than GS and RGS methods whose spectral radius are 0.3643 and 0.1327 , respectively. The exact solution of the system is $(0.2948,0.0932$, $0.0282,0.0861,0.0497,0.0195)$. From table 10 one can see that the solution of the system can be obtained at $9^{\text {th }}$ iteration by using GS method, $5^{\text {th }}$ iteration by using RGS method and $3^{\text {rd }}$ iteration by using SRGS method.Rate of convergence for GS, RGS and SRGS are 1.0098, 2.0197 and 3.0303, respectively. 
Table 8. Numerical results of example 4 and comparison between GS, RGS and SRGS.

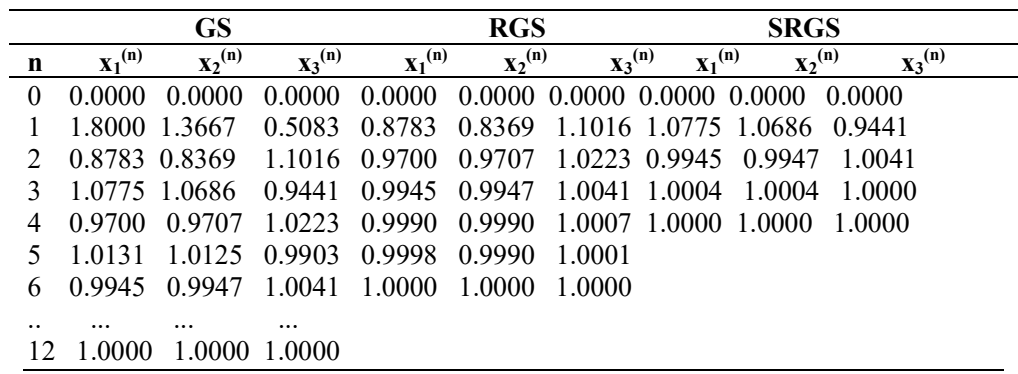

\section{RESULTS AND DISCUSSION}

As presented in Table 1, the spectral radius of SRGS method is smaller than spectral radius of GS and RGS methods, which means that the rate of convergence of SRGS method is larger than rate of convergence of GS and RGS methods. From Table 2, one can also see that the number of iterations of SRGS method is one-third of the number of iterations of GS method and two-third of number of iterations of RGS method. Similarly, Tables 3, 5, 7, and 9 show us the spectral radius of SRGS method is smaller than the spectral radius of GS and RGS methods. Thus, the rate of convergence of SRGS has larger value than rate of convergence of GS and RGS methods. Tables 4, 6, 8 and 10 show us the number of iterations of SRGS method is almost one-third of the number of iterations of GS method and two-third of the number of iterations of RGS method. Theorem 4 shows that SRGS method is better than GS and RGS methods.

\section{CONCLUSION}

Obtaining the solution of a linear system of equations using numerical methods is one of the most important subjects in Applied Science, Engineering and other Sciences. In this paper, we modified refinement of Gauss-Seidel as we call it second-refinement of Gauss-Seidel iterative method for solving linear system of equations which minimizes the number of iterations of GS and RGS methods. Its number of iterations is almost two-third of the number of iterations of RGS method and one-third of number of iterations of GS method need. It has also fast rate of convergence than RGS and GS methods. The result was obtained by MATLAB codes by R2013a (8.1.0.604) 32-bits. A number of theorems have been considered to show the convergence of SRGS method. It is observed that the modified method we presented gives a result faster than both GS \& RGS. For verification, we also compared our results by taking a similar example considered by researchers as shown in example 5. 
Table 10. Numerical results of example 5 and comparison between GS, RGS and SRGS.

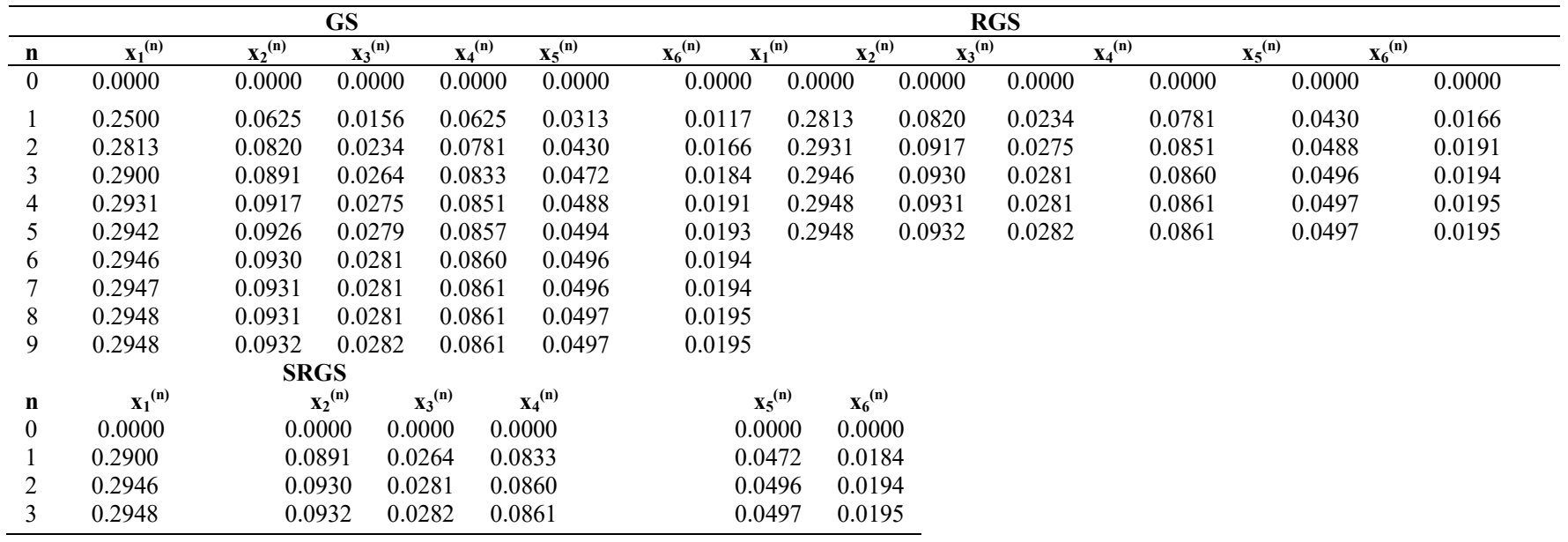




\section{ACKNOWLEDGEMENTS}

The authors would like to thank the anonymous referees for their valuable comments and suggestions, which actually stimulated this work.

\section{REFERENCES}

Dafchahi, F.N. (2008). A new refinement of Jacobi method for solution of linear system equations $\mathrm{AX}=\mathrm{b}$. International Journal of Contemporary Mathematical Sciences 3(17): 819-827.

Datta, B.N. (2010). Numerical Linear Algebra and Application, Society for Industrial and Applied Mathematics, USA.

Laskar, A.H and Behera, S. (2014a). A new refinement of generalized Gauss-Seidel method for solving system of linear equations. International Journal of Mathematics Archive-5 6(5): 104-108.

Laskar, A.H and Behera, S. (2014b). Refinement of iterative methods for the solution of system of linear equations $\mathrm{Ax}=\mathrm{b}$. IOSR Journal of Mathematics (IOSR-JM) 10 (3): 70-73.

Quarteroni, A., Sacco, R and Saleri, F. (2000). Numerical Mathematics, SpringerVerlag New York, Inc., USA.

Saad, Y. (2003). Iterative methods for sparse linear systems, Society for Industrial and Applied Mathematics, USA.

Salkuyeh, D.K. (2007). Generalized Jacobi and Gauss-Seidel methods for solving linear system of equations. A Journal of Chinese Universities (English Series) 16 (2): 164116.

Varga, R.S. (1962). Matrix iterative analysis, Springer series in computational mathematics, Prentice-Hall, Inc, Englewood Cliffs, New Jersey.

Vatti, V.B.K and Genanew Gofe Gonfa (2011). Refinement of generalized Jacobi (RGJ) method for solving System of Linear Equations. International Journal of Contemporary Mathematical Sciences 6(3): 109-116.

Vatti, V.B.K and Tesfaye Kebede Enyew (2011). A refinement of Gauss-Seidel method for solving of linear system of equations. International Journal of Contemporary Mathematical Sciences 6(3): 117-121. 